\title{
Simultaneous Infection with Shigella sonnei and Vibrio cholerae in a Young Child
}

\author{
LISA H. HOCHSTEIN
}

\begin{abstract}
A 20-month old infant presented to the emergency department with diarrhea and vomiting of six days duration. Blood, stool and urine specimens were collected for both bacteriological culture and parasitic workup. Concurrent infections with both Shigella sonnei and Vibrio cholerae were determined to be the cause of the infectious diarrhea. This case illustrates the importance of the microbiology laboratory in detecting infrequently seen enteric pathogens. We will also review the pathogenesis and laboratory diagnosis of Vibrio cholerae and Shigella sonnei.
\end{abstract}

ABBREVIATIONS: SBAP - sheep blood agar plate, $\mathrm{CBC}$ - complete blood count, IV - intravenous, $\mathrm{NaCl}$ - sodium chloride, Spp. - species, BCG - bacillie Calmette-Guerin, TSI - triple sugar iron agar, LIA lysine iron agar, ADP - adenosine dinucleotide phosphate, cAMP - cyclic adenosine monophosphate, TCBS - thiosulfate citrate bile salts sucrose, RBC - red blood cell, BUN - blood urea nitrogen, $\mathrm{Na}$ - sodium, $\mathrm{K}$ - potassium, $\mathrm{Cl}$ - chloride, $\mathrm{CO}_{2}$ - carbon dioxide, $\mathrm{Ca}$ - calcium, TMP/SMX - trimethoprim/sulfamethoxazole

INDEX TERMS: Shigellosis, cholera, diarrheal illness, gastroenteritis

\section{Clin Lab Sci 2013;26(4):165}

Lisa H. Hochstein, M.S., MLS (ASCP) ${ }^{C M}$, Clinical Laboratory Sciences Program, Department of Pharmacy Administration and Allied Health Sciences, College of Pharmacy and Health Sciences, St. John's University, Queens, NY

Address for Correspondence: Lisa H. Hochstein, M.S., MLS (ASCP) $)^{C M}$, Assistant Professor and Program Director, Clinical Laboratory Sciences Program Department of Pharmacy Administration and Allied Health Sciences, College of Pharmacy and Health Sciences,
St. John's University, Queens, NY, 11365, 718-9908449, hochstel@stjohns.edu

\section{Case Presentation}

A twenty month old female was admitted to a local community hospital. The patient had arrived in the United States from Pakistan four days prior to admission. The chief complaint was diarrhea and vomiting for six days duration. Blood, stool and urine specimens were collected for both bacteriologic and parasitic testing. Other laboratory tests sent on admission included a complete blood count (CBC) with differential, urinalysis and a basic metabolic panel. The patient was also sent for a chest radiograph.

\section{Laboratory Results}

The results of the CBC drawn on admission are shown in Table 1. The differential indicated an increase of segmented neutrophils and bands suggesting the possibility of an infection. The basic metabolic panel initially drawn was hemolyzed. The results of this specimen drawn on admission are shown in Table 2 . Urinalysis also taken on admission showed results within normal reference intervals with a trace of protein and bacteria. The RBC morphology showed microcytosis and hypochromia. The $\mathrm{CBC}$ results indicated a microcytic hypochromic anemia. This child, when admitted, was underweight for her age and exhibited pallor of the skin. The patient was likely nutritionally deficient based on physical examination. The chemistry results indicated an electrolyte imbalance secondary to her gastroenteritis. The physician attending the child thought that the child may have cholera based on her country of origin, symptoms and chemistry results.

Based on these laboratory results taken on admission, the patient was started on IV fluids of $0.9 \% \mathrm{NaCl}$ for dehydration and given iron syrup for low blood hemoglobin, B-complex to aid in carbohydrate digestion and lysine to stimulate the synthesis of 


\section{CLINICAL PRACTICE}

digestive enzymes.

Results of the chest radiograph showed right upper lobe pneumonia. A possibility existed that the patient might have been exposed to tuberculosis. Since the child had received the BCG vaccine while in Pakistan, acid fast cultures were ordered. Administration of the BCG vaccine causes a positive PPD skin test. Therefore, a PPD skin test was not performed. Due to the age of the child and to rule out the possibility of tuberculosis, three gastric aspirate specimens were sent for acid fast culture. All cultures were negative after 6 weeks incubation.

Table 1. Complete Blood Count (CBC) Results

\begin{tabular}{lcc}
\hline $\begin{array}{l}\text { Test } \\
\text { White blood cell }\end{array}$ & $\begin{array}{c}\text { Result } \\
\text { count (WBC) }\end{array}$ & $\begin{array}{c}\text { Reference Values } \\
4.6 \times 10^{9} / \mathrm{L}\end{array}$ \\
$\begin{array}{l}\text { Red blood cell } \\
\text { count (RBC) }\end{array}$ & $3.75 \times 10^{12} / \mathrm{L}$ & $4.1-5.3 \times 10^{12} / \mathrm{L}$ \\
$\begin{array}{l}\text { Hemoglobin } \\
\text { Hematocrit }\end{array}$ & $66 \mathrm{~g} / \mathrm{L}$ & $130-140 \mathrm{~g} / \mathrm{L}$ \\
$\begin{array}{l}\text { Mean Corpuscular } \\
\text { Volume }\end{array}$ & $0.271 \mathrm{~L} / \mathrm{L}$ & $0.33-0.41 \mathrm{~L} / \mathrm{L}$ \\
$\begin{array}{l}\text { Mean Corpuscular } \\
\text { Hemoglobin Concentration }\end{array}$ & $27.2 \mathrm{~g} / \mathrm{dL}$ & $32-37 \mathrm{~g} / \mathrm{dL}$ \\
$\begin{array}{l}\text { Mean Corpuscular } \\
\text { Hemoglobin }\end{array}$ & $15.8 \mathrm{pg}$ & $24-30 \mathrm{pg}$ \\
& &
\end{tabular}

Table 2. Hemolyzed Blood Sample Collected on Admission

\begin{tabular}{lll}
\hline \multicolumn{1}{c}{ Test } & Result & Reference Values \\
Blood urea nitrogen $(\mathrm{BUN})$ & $16 \mathrm{mg} / \mathrm{dL}$ & $5-18 \mathrm{mg} / \mathrm{dL}$ \\
Sodium $(\mathrm{Na})$ & $134 \mathrm{mmol} / \mathrm{L}$ & $138-145 \mathrm{mmol} / \mathrm{L}$ \\
Potassium $(\mathrm{K})$ & $3.9 \mathrm{mmol} / \mathrm{L}$ & $3.4-4.7 \mathrm{mmol} / \mathrm{L}$ \\
Chloride $(\mathrm{Cl})$ & $106 \mathrm{mmol} / \mathrm{L}$ & $98-107 \mathrm{mmol} / \mathrm{L}$ \\
Carbon dioxide $(\mathrm{CO} 2)$ & $18 \mathrm{mmol} / \mathrm{L}$ & $20-28 \mathrm{mmol} / \mathrm{L}$ \\
Glucose & $100 \mathrm{mg} / \mathrm{dL}$ & $60-100 \mathrm{mg} / \mathrm{dL}$ \\
Creatinine & $0.4 \mathrm{mg} / \mathrm{dL}$ & $0.3-0.7 \mathrm{mg} / \mathrm{dL}$ \\
Calcium $(\mathrm{Ca})$ & $8.4 \mathrm{mg} / \mathrm{dL}$ & $8.6-10.2 \mathrm{mg} / \mathrm{dL}$
\end{tabular}

Blood cultures drawn on admission showed no growth after 5 days incubation. The urine culture showed mixed growth after 24 hours incubation indicating the specimen was contaminated. The stool specimen sent for rotavirus testing was negative and the testing for ova and parasites was not performed due to improper collection of the specimen. The first stool specimen collected on admission was cultured according to the laboratory's protocol. This protocol included the use of the following media: MacConkey, Hektoen Enteric,
Salmonella Shigella and Campylobacter with 5 antimicrobials (amphotericin B, cephalothin, trimethoprim, vancomycin and polymyxin B) and 10\% sheep blood (Campy-BAP) agars (all from Becton Dickinson (BD) Diagnostics, Sparks, MD). Results of the culture indicated no Salmonella, Shigella, Campylobacter or Yersinia spp. isolated. On the second day of admission, a new stool specimen was sent due to continued diarrhea.

This second specimen was cultured again according to the laboratory's protocol. Nonlactose fermenting colonies were noted to be growing on both the Hektoen Enteric and Salmonella Shigella agars. Along with a SBAP, three screening tubed media, (TSI, LIA and urea agar) (all from BD Diagnostics, Sparks, MD) were inoculated. The SBAP was inoculated for use with the oxidase test and any serological grouping that might be necessary based on the results of the three screening media. Results are shown in Table 3. These results suggested the possibility of Shigella. Using the inoculum from the SBAP, the isolate was serologically grouped using Shigella antisera. Results are shown in Table 4. Preliminary results were reported. The isolate was placed in the Vitek 2 (bioMerieux, Durham, NC) instrument for definitive biochemical identification and antibiotic susceptibility testing. Biochemical results confirmed the presence of Shigella sonnei. The confirmatory results and susceptibility profile were reported. As per laboratory protocol, the Gas Pak jar (BD Diagnostics, Sparks, MD) containing a Campy Pak (BD Diagnostics, Sparks, MD) was opened after 48 hours of incubation. Growth was noted on the CampyBAP plate. A Gram stain and an oxidase test were performed. The organism on the plate did not resemble Campylobacter, even though the morphology indicated Gram negative comma shaped bacilli and the oxidase test was positive. A biochemical panel using the Vitek 2 was inoculated and placed in the instrument.

At 18 hours of incubation, the profile given by the Vitek 2 indicated the possibility of Vibrio cholerae, but the level of identification was not high. To confirm the presence of Vibrio cholerae the isolate was set up on API 20E strips (bioMerieux, Durham, NC) in duplicate, one using $0.85 \% \mathrm{NaCl}$ for the inoculum suspension and the other using sterile distilled water. This was done to establish the halophilic nature of the organism. After overnight incubation, the API 20E strip 


\section{CLINICAL PRACTICE}

that was inoculated using saline gave a very good identification of Vibrio cholerae. The API 20E strip that used distilled water gave an identification of "good likelihood, low selectivity" with Vibrio cholerae being one of the possible organisms. An identification of presumptive Vibrio cholerae was made. This information was also reported. The isolate was subsequently sent to the New York City Department of Health for typing. Typing indicated the isolate was Vibrio cholerae serotype 01.

Table 3. Stool Culture Screening Results

\begin{tabular}{cl}
\hline TSI & Alkaline/Acid; no gas or H2S observed \\
LIA & Alkaline/Acid; no H2S observed \\
Urea Agar & Negative (no color change) \\
\hline
\end{tabular}

Table 4. Results of Shigella Grouping

$\begin{array}{ll}\text { Shigella Group A } & \text { Negative } \\ \text { Shigella Group B } & \text { Negative } \\ \text { Shigella Group C } & \text { Negative } \\ \text { Shigella Group D } & \text { Positive }\end{array}$

The laboratory performed susceptibility testing on the presumptive Vibrio cholerae isolated using the standard Kirby Bauer disk diffusion method. These results for both Shigella sonnei and Vibrio cholerae are shown in Table 5.

Table 5. Susceptibility Results for Shigella sonnei and Vibrio cholerae

\begin{tabular}{lll}
\hline Antibiotic & Shigella sonnei & Vibrio cholerae \\
Ampicillin & Susceptible & Susceptible \\
TMP/SMX & Susceptible & Resistant \\
Ciprofloxacin & Susceptible & Susceptible \\
Ceftiaxone & Susceptible & \\
Ampicillin/Sulbactam & Susceptible & \\
Ticarcillin/Clavulanate & Susceptible & \\
& & \\
\hline
\end{tabular}

\section{Pathogenesis of Vibrio cholerae}

Vibrio cholerae 01 is the causative agent of Asiatic cholera. Vibrio species are free-living bacteria found in aquatic environments throughout the world. They tend to be more common in warmer waters. Epidemic strains of $\mathrm{V}$. cholerae that carry specific virulence genes cause the disease cholera. ${ }^{1} \mathrm{~V}$. cholerae 01 may be subdivided by biotype: El Tor (the most common biotype) or classic; by serotype: Inaba, Ogawa or Hikojima; or by toxin production: toxigenic or nontoxigenic. ${ }^{2}$ This agent was first described in 1854 in Italy by Filippo Pacini, who discovered large numbers of curved bacteria in the intestinal contents of cholera victims. ${ }^{3}$ At about the same time as Pacini, John Snow in London showed that cholera was associated with consumption of water from a water system that obtained its water from the Thames River at a point below major sewage inflows. ${ }^{4} \mathrm{~V}$. cholerae was also characterized by Robert Koch. It was his work on cholera that led the way to establishing the theory of disease and helped convince the medical community as to the nature of this clinical condition. ${ }^{5}$ Koch also proposed that the agent responsible for cholera produced "a special poison" which acted on the intestinal epithelium and that the symptoms of cholera could be "regarded essentially as a poisoning". ${ }^{4}$ This disease has been, and still remains, a major illness of public health significance in developing areas of the world. Today Vibrio infections are becoming increasingly common in the United States. ${ }^{2}$ Therefore, cholera should not be disregarded as a diagnostic possibility in patients with diarrhea.

Most cases of cholera in the United States are acquired by those residing in endemic areas who immigrate to the United States, through foreign travel to areas of poor sanitation and questionable drinking water. Cholera may also be acquired through eating raw or improperly cooked seafood coming from the Gulf Coast where a particular Vibrio cholerae 01 (biotype El Tor, serotype Inaba) is endemic. ${ }^{2}$ It is important that clinical laboratory personnel always be aware of the fact that an unusual organism may find its way into the daily work of a clinical microbiology laboratory.

Cholera is a potentially epidemic and life-threatening secretory diarrhea characterized by numerous, voluminous watery stools often accompanied by vomiting which may progress to hypovolemic shock and acidosis. Cholera is usually spread through contaminated water. It is seen in areas where the level of sanitation is poor. Drinking water is often identified as the primary vehicle for transmission. Even in areas where access to safe water is available, interruption by natural disaster may also compromise safe drinking water. Endemic diarrheal disease outbreaks can occur after drinking water has been contaminated. This can occur after flooding and related displacement. ${ }^{6}$ Cholera spreads in both endemic and epidemic patterns by the ingestion of the toxigenic organisms. Contaminated 


\section{CLINICAL PRACTICE}

food has also been noted in the transmission of cholera. Foods may be contaminated by food handlers during or after preparation. Fruits and vegetables sprayed with water to maintain "freshness" may acquire cholera organisms. Lastly, human fertilizers may also contaminate fruits or vegetables. ${ }^{7}$

Risk factors for cholera infections/outbreaks include the size of the inoculum ingested. This is directly related to the likelihood of developing illness. Large inocula $\left(10^{6}\right.$ $10^{11}$ colony-forming units) are required to produce severe disease because gastric acid decreases the viability of the organism. ${ }^{7,8} \mathrm{~A}$ rare risk factor for cholera is blood group antigen $\mathrm{O}$. Individuals with blood type $\mathrm{O}$ are substantially more likely to contract cholera and it is more likely to be severe during El Tor epidemics. The molecular basis for this is not known. ${ }^{7}$

The symptoms of this disease include severe gastroenteritis along with vomiting and diarrhea. Stool samples produced in cholera are described as "rice water". This description is relative to the appearance of watery specimens with mucus flecks. Stool may be produced as many as 10-30 times per day. The clinical presentation of cholera is due to the organism's ability to produce a powerful enterotoxin called a choleragen or cholera toxin. After the organisms are ingested and survive the acid environment of the stomach, they will quickly colonize and multiply in the small intestine and begin producing the cholera toxin. The toxin is a hetero-oligomer consisting of five $\mathrm{B}$ subunits and a single A subunit. ${ }^{8}$

The B subunit is used for specific binding to the $\mathrm{GM}_{1}$ ganglioside receptor which is located on the surface of the epithelial cells that line the intestinal mucosa. Upon binding, the A subunit is translocated into the host cell cytosol where it is activated by thiol dependent reduction. The resulting nicked $A_{1}$ subunit possesses an adenosine dinucleotide phosphate (ADP)-ribosylating activity that targets the host cell G-protein. ADPribosylated $G$ protein permanently activates adenylate cyclase activity leading to increased levels of intracellular cyclic adenine monophosphate (cAMP). When activated, cAMP inhibits active sodium chloride absorption and increases chloride and bicarbonate secretion. As a result of this activity, there is passive water loss leading to a marked decrease in intravascular volume, hypotension and hypoperfusion of critical organs. ${ }^{8}$ In large numbers of cases of cholera, the volume of water lost can be so great that the patient is rapidly dehydrated and may go into shock. If not quickly treated with IV fluids, the patient may die. Plain salt water is non-absorbable during cholera and aggravates diarrhea. The addition of glucose renders the solution absorbable and aids in providing oral rehydration. ${ }^{1}$ This toxin-mediated illness causes colonization rather than penetrating the mucosal barrier of the intestine. ${ }^{7}$ Due to these factors, blood and inflammatory cells are absent in cholera. We see instead the "rice water" stools which is typical of cholera toxin activity.

\section{Pathogenesis of Shigella sonnei}

Shigellae are Gram negative, facultative anaerobic bacilli that are members of the family Enterobacteriaceae. They are the causative agent of shigellosis or bacillary dysentery. Bacillary dysentery was first differentiated from amoebic dysentery in 1887. Its etiologic agent, Bacillus dysenteriae was isolated and described by Shiga in $1898 .{ }^{10}$ This invasive infection of the colon can present with symptoms ranging from short-lasting watery diarrhea to acute inflammatory bowel disease. In the classic infection, it is characterized by fever, intestinal cramps and bloody diarrhea with mucopurlent stool. ${ }^{11}$

In developing countries, shigellosis is most common in children under the age of 5 years old. It is usually spread by the excreta of infected individuals either directly by the fecal-oral route or by contaminated food or water. Overcrowding and water supplies inadequately protected from sewage contamination contribute to the high incidence of infection. ${ }^{10}$

Shigellosis is a highly contagious disease. Ingestion of as few as 10 organisms can cause disease. Shigella species are relatively resistant to stomach acid and may allow survival of small numbers of ingested organisms providing them the opportunity to infect the intestinal mucosa.

Once the intestinal mucosa is penetrated, the Shigella infection is characterized by the degeneration of the epithelium followed by an acute inflammatory colitis in the lamina propria. This ultimately leads to desquamation and ulceration of the mucosa causing leakage of blood, inflammatory elements and mucus into the intestinal lumen. ${ }^{10}$ 


\section{CLINICAL PRACTICE}

\section{Laboratory Diagnosis of Vibrio cholerae}

Vibrios may grow on blood and MacConkey agars, but isolation is enhanced by using thiosulfate citrate bile salts sucrose (TCBS) agar. On this medium, Vibrio cholerae appears as yellow colonies due to the fermentation of sucrose. ${ }^{7}$ Identification of Vibrio cholerae can be problematic. This organism's identification using semi-automated as well as automated methods available cannot always give the correct answer. Sometimes our testing results in an answer of "low discrimination" requiring supplemental testing which may not be readily available in every laboratory.

Vibrio cholerae is a gram-negative, curved bacillus motile by means of a single polar flagellum. This organism can be identified both biochemically and serologically. It is characterized by a positive oxidase reaction which aids in differentiating it from members of the Enterobacteriaceae. Vibrio cholerae is able to ferment both glucose and sucrose but not lactose. The organism is also able to decarboxylate the amino acids lysine and ornithine but not arginine. It is also able to breakdown tryptophan to produce indole.

Serologic testing can also be used for identification of this organism. In the United States, the use of antisera is not commonly used due to the infrequent isolation of the organism. This type of testing is usually performed by state and public health laboratories.

Lastly, a diagnosis of cholera can be made serologically with the patient demonstrating evidence of serologic conversion. A vibriocidal antibody titer of greater than 1:640 suggests recent infection. Serologic diagnosis may also be made by a four-fold increase in titers two weeks after exposure and a decrease in titers two months after exposure. $^{2}$

\section{Laboratory Diagnosis of Shigella sonnei}

Shigellae can be identified on the basis of several biochemical characteristics. These organisms do not ferment lactose but do ferment glucose with production of acid but no gas. They do not produce hydrogen sulfide. Shigellae are consistently nonmotile due to their lack of flagella. Shigella sonnei are biochemically different from the other Shigella species. Shigella sonnei possess both ornithine decarboxylase and $\beta$ galactosidase activity.
Shigella can also be identified serologically. Four serogroups (serogroup A-D) are known, each corresponding to a specific species. Shigella sonnei are members of serogroup D.

\section{DISCUSSION}

The clinical microbiology laboratory is often called upon to aid in the diagnosis of an enteric infection. In most cases, usual enteric pathogens are suspected as the causative agent. These include such organisms as Salmonella, Shigella and Campylobacter. Sometimes, if necessary, we will be advised by the physician to examine the culture for other pathogens such as E. coli O157:H7, Yersinia or Aeromonas.

This particular case illustrates two important points. First, all medical laboratory scientists must be aware of and keep up-to-date about unusual organisms seldom seen. Since people come to the United States from all over the world, clinical microbiology laboratories must be prepared for even rarely seen organisms. Also, in this age of travel to more exotic places, returning vacationers, business travelers, etc. can also come home infected by these organisms. As unusual organisms such as Vibrio cholerae are rarely seen, it is even more important to recognize them when encountered.

Second, the clinical microbiology laboratory must examine the culture and look for any and all potential pathogens that may be present. In this case presentation, an enteric pathogen, Shigella sonnei, was initially isolated from the patient. It is a reasonable explanation why the patient presented with gastroenteritis. Correlating clinical history including the country where the patient was from, plus the symptoms presented, cholera was reasonable as part of the differential diagnosis. The fact that the medical laboratory scientist continued to investigate the organism growing on the Campy-BAP agar, even after the isolation and identification of Shigella sonnei, allowed the correct diagnosis of cholera to be determined. It is entirely possible for a patient to arrive at the hospital with more than one organism causing their symptoms. Given the fact that the patient was from a country with poor sanitation, multiple enteric organisms were a possibility.

\section{REFERENCES}

1. Morris, Jr.,JG. Cholera and other types of vibriosis: A story of human pandemics and oysters on the half shell. Clin. Infect. 


\section{CLINICAL PRACTICE}

Dis. 2003;37:272-9.

2. Daniels, NA and Shafaie, A. A review of pathogenic Vibrio infections for clinicians. Infect. Med. 2000;17:665-85.

3. Bentivoglio, M and Pacini, P. Filippo Pacini: A determined observer. Brain Res. Bulletin. 1995;38:161-5.

4. Kaper, JB, Morris,Jr., JG and Levine, MM. Cholera. Clin. Microbiol. Rev. 1995;8:48-86.

5. Howard-Jones, N. Robert Koch and the cholera vibrio: A centenary. Br. Med. J. 1984;288:379-81.

6. Watson, JT, Gayer, M and Connolly, MA. Epidemics after natural disasters. Emerg. Infect. Dis. 2007;13:1-5.

7. Lacey, SW. Cholera: Calamitous past, ominous future. Clin. Infect. Dis. 1995;20:1409-19.
8. Reidl, J and Klose, KE. Vibrio cholerae and cholera: Out of the water and into the host. FEMS Microbiol. Rev. 2002;26:12539.

9. Hale, TL. Genetic Basis of Virulence in Shigella Species. Microb. Rev. 1991;55:206-24.

10. Sansonetti, PJ. Microbes and Microbial Toxins: Paradigms for Microbial-Mucosal Interactions, III. Shigellosis: from symptoms to molecular pathogenesis. Am. J. Physiol. Gastrointest. Liver Physiol. 2011;280:G319-23.

11. O'Hara, CM, Sowers, EG, Bopp, CA, Duda, SB and Strockbine, NA. Accuracy of six commercially available systems for identification of members of the family Vibrionaceae. J. Clin. Microb. 2003;41:5654-9.

The peer-reviewed Clinical Practice Section seeks to publish case studies, reports, and articles that are immediately useful, are of a practical nature, or contain information that could lead to improvement in the quality of the clinical laboratory's contribution to patient care, including brief reviews of books, computer programs, audiovisual materials, or other materials of interest to readers. Direct all inquiries to Perry Scanlan, PhD, MT(ASCP), Medical Technology, Austin Peay State University, Room D212, Sundquist Science Complex, Box 4668, Clarksville TN 37044. Clinical Laboratory Science encourages readers to respond with thoughts, questions, or comments regarding these articles. Email responses to westminsterpublishers@comcast.net. In the subject line, please type the journal issue and lead author such as "CLIN LAB SCI 26(4) RE HOCHSTEIN". Selected responses may appear in the Dialogue and Discussion section in a future issue. Responses may be edited for length and clarity. We look forward to hearing from you. 